\title{
Half-exact coherent functors over Dedekind domains
}

Adson Banda

The self-archived postprint version of this journal article is available at Linköping University Institutional Repository (DiVA):

http://urn.kb.se/resolve?urn=urn:nbn:se:liu:diva-158347

N.B.: When citing this work, cite the original publication.

Banda, A., (2019), Half-exact coherent functors over Dedekind domains, Journal of Algebra and its Applications, 18(5), 1950099. https://doi.org/10.1142/So219498819500993

Original publication available at:

https://doi.org/10.1142/So219498819500993

Copyright: World Scientific Publishing

http://www.worldscientific.com/ 


\title{
HALF EXACT COHERENT FUNCTORS OVER DEDEKIND DOMAINS
}

\author{
ADSON BANDA \\ Department of Mathematics, Linköping University, Sweden \\ and \\ Department of Mathematics and Statistics, University of Zambia \\ adson.banda@liu.se
}

\begin{abstract}
Let $A$ be a principal ideal domain (PID) or more generally a Dedekind domain and let $F$ be a coherent functor from the category of finitely generated $A$-modules to itself. We classify the half exact coherent functors $F$. In particular, we show that if $F$ is a half exact coherent functor over a Dedekind domain $A$, then $F$ is a direct sum of functors of the form $\operatorname{Hom}_{A}(P,-), \operatorname{Hom}_{A}\left(A / \mathfrak{p}^{s},-\right)$ and $A / \mathfrak{p}^{s} \otimes-$, where $P$ is a finitely generated projective $A$-module, $\mathfrak{p}$ a non-zero prime ideal in $A$ and $s \geq 1$.
\end{abstract}

\section{InTRODUCTION AND NotATION}

Since the foundational work of Maurice Auslander [1] and the more specialized work of Robin Hartshorne [5], coherent functors have proved to be a useful tool in understanding and describing properties of some mathematical objects. For instance, they were used to describe infinitesimal deformation theory by Michael Schlessinger [10] and also to describe Artin's criteria for algebraicity of a stack by Jack Hall [4]. Recently, Gustav Sædén Ståhl [11] used coherent functors to study properties of Rees algebras of modules.

Other authors who have worked with coherent functors include: Vincent Franjou and Teimuraz Pirashvili [3] who linked coherent functor to strict polynomial functors, David B. Jaffe [6] who applied coherent functors to torsion in the Picard group, and Henning Krause [7] who studied a class of what he called a definable subcategory which arises from a family of coherent functors $F_{i}$ on an abelian category $\mathcal{A}$ by taking all objects $X$ in $\mathcal{A}$ such that $F_{i}(X)=0$ for all $i$.

In [1], Auslander introduced coherent functors on abelian categories. Here, like in [5], we study coherent functors on the category of finitely generated modules over a commutative noetherian ring. 
Hartshorne [5, 6.1] classified half exact coherent functors over a discrete valuation ring (DVR). The purpose of this paper then is to extend this classification of half exact coherent functors on finitely generated modules to principal ideal domains (PIDs) and more generally to Dedekind domains. We also extend [5, 3.5]. Our approach in both cases is similar to that of Hartshorne's [5].

We now fix some notation. In this paper, all rings $A$ are assumed to be commutative and noetherian, and all functors $F$ are assumed to be covariant and $A$-linear. We will denote by $\mathcal{M}_{A}^{f g}$ the category of finitely generated $A$-modules. The category of covariant $A$-linear functors from $\mathcal{M}_{A}^{f g}$ to itself will be denoted by $\mathcal{F}_{A}^{f g}$. For $M \in \mathcal{M}_{A}^{f g}$ we denote by $h_{M}$ the functor $\operatorname{Hom}_{A}(M,-) \in \mathcal{F}_{A}^{f g}$.

Section 2 recalls the definition and some properties of functors mainly found in [1] and [5]. At the end of the section, we extend Proposition 3.5 in [5]. Precisely, we show that if $F: \mathcal{M}_{A}^{f g} \rightarrow \mathcal{M}_{A}^{f g}$ is coherent, then the natural map $\widehat{F(M)} \rightarrow \underset{\leftarrow}{\lim } F\left(M / \mathfrak{a}^{n} M\right)$ is an isomorphism for any $M \in \mathcal{M}_{A}^{f g}$ and any ideal $\mathfrak{a}$ of $A$ with

$$
\operatorname{Supp}(M / \mathfrak{a} M) \subset \operatorname{Max} A .
$$

Here, $\operatorname{Max} A$ is the set of all maximal ideals in $A$ and $\widehat{F(M)}$ is the completion of $F(M)$ in the $\mathfrak{a}$-adic topology.

In section 3 , we devote ourselves to the construction of a functor $\tilde{F}$. For $S \subset A$ a multiplicative subset and $F \in \mathcal{F}_{A}^{f g}$, we use the fact that every finitely generated $S^{-1} A$-module is isomorphic to $S^{-1} N$ for some $A$-module $N$ to define $\tilde{F}$ on finitely generated $S^{-1} A$-modules. We then show that $\tilde{F}$ is left-, right- or half-exact whenever $F$ is left-, right- or half-exact. It also turns out that $\tilde{F}$ is coherent whenever $F$ is. When $S=A \backslash \mathfrak{p}$, we denote $\tilde{F}$ by $F_{\mathfrak{p}}$. This is used in the proof of 4.5 . The functor $\tilde{F}$ also appears in [5] in a more general setting.

In section 4 , we turn to the main purpose of this paper. We consider the exact sequence

$$
F(A) \otimes-\stackrel{\alpha}{\longrightarrow} F \longrightarrow F_{0} \longrightarrow 0
$$

and show that $\alpha_{M}: F(A) \otimes M \rightarrow F(M)$ is injective for any $A$-module $M$ with projective dimension at most 1 . We then show that if $F$ is a half exact coherent functor over a PID, then $F_{0}$ in (1) is left exact. In this case $F_{0} \cong \operatorname{Hom}_{A}(N,-)$ for some finitely generated module $N$. We further show that over a PID, the sequence (1) splits, that is, $F \cong F(A) \otimes-\oplus F_{0}$. From this, we deduce that $F$ is a direct sum of the identity functor, $\operatorname{Hom}_{A}\left(A / p^{s},-\right)$ and $A / p^{s} \otimes-$, where $s \geq 1$ and $p \in A$ is irreducible.

When $A$ is a Dedekind domain, by localizing to prime ideals, we show that $F_{0}$ is left exact and the sequence (1) splits. Hence, we deduce that $F$ is a direct sum of $\operatorname{Hom}_{A}(P,-), \operatorname{Hom}_{A}\left(A / \mathfrak{p}^{s},-\right)$ and $A / \mathfrak{p}^{s} \otimes-$, where 
$P$ is a finitely generated projective $A$-module, $s \geq 1$ and $\mathfrak{p}$ a prime ideal in $A$.

\section{Coherent Functors}

Definition 2.1. Let $A$ be a noetherian ring. A functor $F \in \mathcal{F}_{A}^{f g}$ is said to be coherent if it can be presented as a cokernel of Hom-functors; i.e, if there are $M, N \in \mathcal{M}_{A}^{f g}$ and an exact sequence

$$
h_{N} \rightarrow h_{M} \rightarrow F \rightarrow 0 .
$$

We will denote by $\mathcal{C}_{A}^{f g}$ the full subcategory of $\mathcal{F}_{A}^{f g}$ consisting of all coherent functors on $\mathcal{M}_{A}^{f g}$.

It is known, for instance $[5,1.1]$, that $\mathcal{C}_{A}^{f g}$ is closed under extensions, kernels and cokernels. That is, if $0 \rightarrow F \rightarrow H \rightarrow G \rightarrow 0$ is an exact sequence in $\mathcal{C}_{A}^{f g}$ with $F$ and $G$ in $\mathcal{C}_{A}^{f g}$, then $H \in \mathcal{C}_{A}^{f g}$; and if $\tau: F \rightarrow G$ is a morphism in $\mathcal{C}_{A}^{f g}$, then $\operatorname{Ker} \tau$, Coker $\tau$ and $\operatorname{Im} \tau$ are also in $\mathcal{C}_{A}^{f g}$.

Now, let $F$ be any functor in $\mathcal{F}_{A}^{f g}$. It is well known (Eilenberg-Watts theorem) that there is a natural map $\alpha: F(A) \otimes-\rightarrow F$. Clearly, $F$ is right exact if $\alpha$ is a natural isomorphism. The converse is also true. In particular, $F$ is right exact if and only if it has the form $M \otimes-$ for some $M \in \mathcal{M}_{A}^{f g}$. Furthermore, if $F_{0}=\operatorname{Coker} \alpha$, then $F_{0}$ satisfies the following:

i) $F_{0}(A)=0$,

ii) $F_{0}$ is coherent if $F$ is coherent, and

iii) $F_{0}$ is half exact if $F$ is half exact.

Thus we have an exact sequence

$$
F(A) \otimes-\stackrel{\alpha}{\longrightarrow} F \longrightarrow F_{0} \longrightarrow 0 .
$$

Evidently, $\alpha_{L}$ is an isomorphism for every finite free $A$-module $L$.

Proposition 2.2. For all projective modules $P$ in $\mathcal{M}_{A}^{f g}$, the natural map $\alpha_{P}: F(A) \otimes P \rightarrow F(P)$ is an isomorphism.

Proof. Let $P$ be a projective module in $\mathcal{M}_{A}^{f g}$. Then, there is a surjective homomorphism $f: L \rightarrow P$ where $L$ is a finite free $A$-module. Since $P$ is projective, there exists a homomorphism $g: P \rightarrow L$ such that $f \circ g=1_{P}$. Hence we have $1_{F(P)}=F(f \circ g)=F(f) \circ F(g)$.

Now consider the commutative diagram.

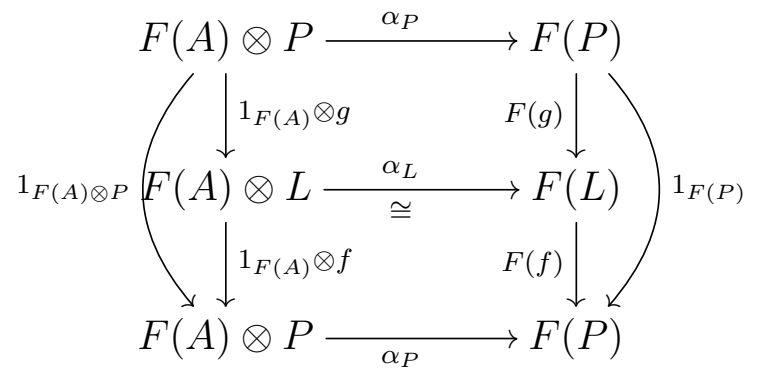


Then we have

$$
\begin{aligned}
\alpha_{P} \circ\left[\left(1_{F(A)} \otimes f\right) \circ \alpha_{L}^{-1} \circ F(g)\right] & =F(f) \circ F(g)=1_{F(P)} \text { and } \\
{\left[\left(1_{F(A)} \otimes f\right) \circ \alpha_{L}^{-1} \circ F(g)\right] \circ \alpha_{P} } & =\left(1_{F(A)} \otimes f\right) \circ\left(1_{F(A)} \otimes g\right) \\
& =1_{F(A) \otimes P .}
\end{aligned}
$$

Therefore, $\alpha_{P}$ is an isomorphism.

Corollary 2.3. If $A$ is a semisimple ring, then any functor $F$ in $\mathcal{F}_{A}^{f g}$ is isomorphic to $F(A) \otimes$ - and hence is coherent.

Proof. By [9, 4.5], any module over a semisimple ring is projective and 2.2 applies.

Now, let $A$ be a noetherian ring and let $\mathfrak{a}$ be an ideal in $A$. For every $M$ in $\mathcal{M}_{A}^{f g}$ and $n \geq 1$, there is a natural map $\widehat{F(M)} \rightarrow \lim F\left(M / \mathfrak{a}^{n} M\right)$ which arises by taking the inverse limit of $F(M) / \mathfrak{a}^{n} F(M) \rightarrow F\left(M / \mathfrak{a}^{n} M\right)$.

The next result extends Proposition 3.5 in [5] and our proof follows the proof given there.

Proposition 2.4. Let $A$ be a noetherian ring and let $F$ be in $\mathcal{C}_{A}^{f g}$. Then the natural map $\widehat{F(M)} \rightarrow \underset{\longleftarrow}{\lim } F\left(M / \mathfrak{a}^{n} M\right)$ is an isomorphism for any $M \in \mathcal{M}_{A}^{f g}$ and any ideal $\mathfrak{a}$ of $A$ with

$$
\operatorname{Supp}(M / \mathfrak{a} M) \subset \operatorname{Max} A .
$$

Proof. Let $h_{L} \rightarrow h_{N} \rightarrow F \rightarrow 0$ be a presentation of $F$ as a coherent functor. Since the completion functor is exact, for any $M$ in $\mathcal{M}_{A}^{f g}$, we have an exact sequence

$$
\widehat{h_{L}(M)} \rightarrow \widehat{h_{N}(M)} \rightarrow \widehat{F(M)} \rightarrow 0 .
$$

Also, we have exact sequences

$$
h_{L}\left(M / \mathfrak{a}^{n} M\right) \rightarrow h_{N}\left(M / \mathfrak{a}^{n} M\right) \rightarrow F\left(M / \mathfrak{a}^{n} M\right) \rightarrow 0
$$

which form an exact sequence of inverse systems.

Since $\operatorname{Supp}(M / \mathfrak{a} M) \subset \operatorname{Max} A$, the modules $M / \mathfrak{a}^{n} M$ have finite length. Hence all the modules in (2) are of finite length. Let

$$
J_{n}=\operatorname{Ker}\left(h_{L}\left(M / \mathfrak{a}^{n} M\right) \rightarrow h_{N}\left(M / \mathfrak{a}^{n} M\right)\right)
$$

and let

$$
K_{n}=\operatorname{Ker}\left(h_{N}\left(M / \mathfrak{a}^{n} M\right) \rightarrow F\left(M / \mathfrak{a}^{n} M\right)\right),
$$

then there are short exact sequences

$$
0 \rightarrow J_{n} \rightarrow h_{L}\left(M / \mathfrak{a}^{n} M\right) \rightarrow K_{n} \rightarrow 0
$$

and

$$
0 \rightarrow K_{n} \rightarrow h_{N}\left(M / \mathfrak{a}^{n} M\right) \rightarrow F\left(M / \mathfrak{a}^{n} M\right) \rightarrow 0
$$


of inverse systems. Since for each $n$, the modules $J_{n}$ and $K_{n}$ have finite length, the kernel inverse systems $\left\{J_{n}\right\}$ and $\left\{K_{n}\right\}$ satisfy the MittagLefler condition. By $[8,10.3]$, we have exact sequences

$$
0 \rightarrow \lim _{\longleftarrow} J_{n} \rightarrow \lim _{\longleftarrow} h_{L}\left(M / \mathfrak{a}^{n} M\right) \rightarrow \lim _{\longleftarrow} K_{n} \rightarrow 0
$$

and

$$
0 \rightarrow \lim _{\longleftarrow} K_{n} \rightarrow \lim _{\longleftarrow} h_{N}\left(M / \mathfrak{a}^{n} M\right) \rightarrow \lim _{\longleftarrow} F\left(M / \mathfrak{a}^{n} M\right) \rightarrow 0,
$$

which in turn gives us the desired exact sequence

$$
\lim _{\longleftarrow} h_{L}\left(M / \mathfrak{a}^{n} M\right) \rightarrow \lim _{\longleftarrow} h_{N}\left(M / \mathfrak{a}^{n} M\right) \rightarrow \lim _{\longleftarrow} F\left(M / \mathfrak{a}^{n} M\right) \rightarrow 0 .
$$

Now, we have

$$
\begin{aligned}
\lim _{\leftarrow} h_{N}\left(M / \mathfrak{a}^{n} M\right) & \cong \lim _{\longleftarrow} \operatorname{Hom}_{A}\left(N, M / \mathfrak{a}^{n} M\right) \\
& \cong \operatorname{Hom}_{A}\left(N, \underset{\leftarrow}{\lim } M / \mathfrak{a}^{n} M\right) \\
& \cong \operatorname{Hom}_{A}(N, \widehat{M}) \\
& \cong \operatorname{Hom}_{\hat{A}}\left(N \otimes_{A} \hat{A}, M \otimes_{A} \hat{A}\right) \\
& \cong \hat{A} \otimes_{A} \operatorname{Hom}_{A}(N, M) \\
& \cong \widehat{h_{N}(M)} .
\end{aligned}
$$

Therefore we have the commutative diagram

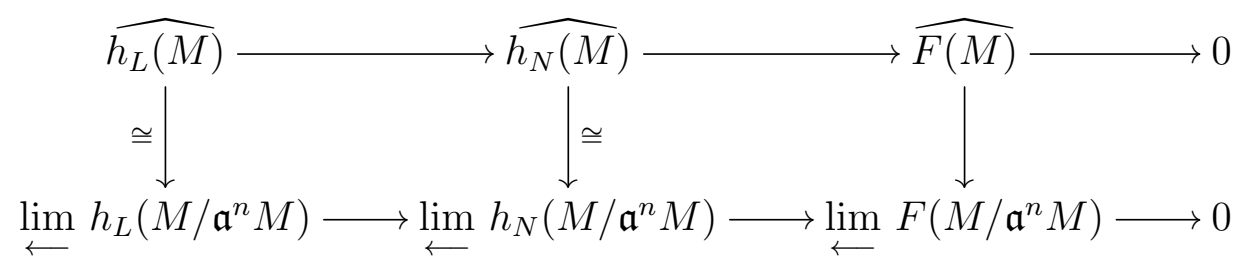

and the five lemma shows that $\widehat{F(M)} \cong \lim _{\longleftarrow} F\left(M / \mathfrak{a}^{n} M\right)$.

\section{LocAlizATiON}

Let $S \subset A$ be a multiplicative set. For each finitely generated $S^{-1} A-$ module $M$ choose one finitely generated $A$-module $N$ together with an isomorphism $\eta_{M}: S^{-1} N \rightarrow M$. If $S^{-1} N=M$, we choose $\eta_{M}=1_{S^{-1} N}$.

Now let $F$ be in $\mathcal{F}_{A}^{f g}$ and $f: M \rightarrow M^{\prime}$ be an $S^{-1} A$-module homomorphism with $M$ and $M^{\prime}$ finitely generated. For chosen isomorphisms $\eta_{M}: S^{-1} N \cong M$ and $\eta_{M^{\prime}}: S^{-1} N^{\prime} \cong M^{\prime}$, consider the diagram

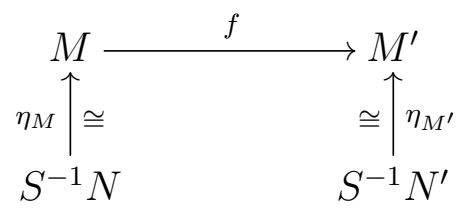


and the natural isomorphism

$$
S^{-1} \operatorname{Hom}_{A}\left(N, N^{\prime}\right) \cong \operatorname{Hom}_{S^{-1} A}\left(S^{-1} N, S^{-1} N^{\prime}\right) .
$$

Define a functor $\tilde{F}$ on objects by $\tilde{F}(M)=S^{-1} F(N)$; and on $S^{-1} A_{-}$ homomorphisms $f$ by

$$
\tilde{F}(f)=\frac{1}{s} S^{-1} F(u)
$$

where $u: N \rightarrow N^{\prime}$ and $s \in S$ are such that $\eta_{M^{\prime}}^{-1} \circ f \circ \eta_{M}=\frac{1}{s} S^{-1} u$.

To show that $\tilde{F}(f)$ is well defined, suppose that $\frac{1}{s} S^{-1} u=\frac{1}{s^{\prime}} S^{-1} u^{\prime}$ for $u, u^{\prime}: N \rightarrow N^{\prime}$ and $s, s^{\prime} \in S$. Then $\frac{1}{s s^{\prime}} S^{-1}\left(s^{\prime} u-s u^{\prime}\right)=0$ and thus, there is $t \in S$ such that $t\left(s^{\prime} u-s u^{\prime}\right)=0$. Since $F$ is $A$-linear, we have

$$
t\left(S^{-1} F\left(s^{\prime} u-s v^{\prime}\right)\right)=S^{-1} F\left(t\left(s^{\prime} u-s u^{\prime}\right)\right)=0
$$

so that $\frac{1}{s} S^{-1} F(u)=\frac{1}{s^{\prime}} S^{-1} F\left(u^{\prime}\right)$. Hence $\tilde{F}(f)$ is well defined.

Next, we show that $\tilde{F}$ is a functor. Let $f: M^{\prime} \rightarrow M$ and $g: M \rightarrow M^{\prime \prime}$ be $S^{-1} A$-homomorphisms. Consider the diagram

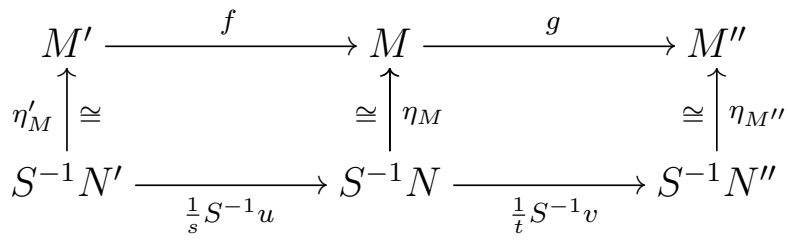

for some $s, t \in S, u: N^{\prime} \rightarrow N$ and $v: N \rightarrow N^{\prime \prime}$ such that $\frac{1}{s} S^{-1} u=$ $\eta_{M}^{-1} \circ f \circ \eta_{M^{\prime}}$ and $\frac{1}{t} S^{-1} v=\eta_{M^{\prime \prime}}^{-1} \circ g \circ \eta_{M}$. Here we have

$$
\left(\eta_{M^{\prime \prime}}^{-1} \circ g \circ \eta_{M}\right) \circ\left(\eta_{M}^{-1} \circ f \circ \eta_{M^{\prime}}\right)=\eta_{M^{\prime \prime}}^{-1} \circ(g \circ f) \circ \eta_{M^{\prime}}
$$

so that $\eta_{M^{\prime \prime}}^{-1} \circ(g \circ f) \circ \eta_{M^{\prime}}=\frac{1}{t s} S^{-1}(v \circ u)$. Thus, we have

$$
\begin{aligned}
\tilde{F}(g \circ f) & =\frac{1}{t s} S^{-1} F(v \circ u) \\
& =\frac{1}{t s} S^{-1}(F(v) \circ F(u)) \\
& =\frac{1}{t} S^{-1} F(v) \circ \frac{1}{s} S^{-1} F(u) \\
& =\tilde{F}(g) \circ \tilde{F}(f)
\end{aligned}
$$

and

$$
\begin{aligned}
\tilde{F}\left(1_{M}\right) & =S^{-1} F\left(1_{N}\right)=S^{-1} 1_{F(N)} \\
& =1_{S^{-1} F(N)}=1_{\tilde{F}(M)} .
\end{aligned}
$$

Therefore, $\tilde{F}$ is an $S^{-1} A$-linear functor on the category of finitely generated $S^{-1} A$-modules.

Remark 3.1. It is clear that $\tilde{F}\left(S^{-1} u\right)=S^{-1} F(u)$. Therefore, if $S^{-1} u$ is an isomorphism, then $S^{-1} F(u)$ is also an isomorphism since functors preserve isomorphisms. 
Theorem 3.2. If $F$ is half exact, then $\tilde{F}$ is also half exact.

Proof. Let $0 \longrightarrow M^{\prime} \stackrel{f}{\longrightarrow} M \stackrel{g}{\longrightarrow} M^{\prime \prime} \longrightarrow 0$ be an exact sequence of finitely generated $S^{-1} A$-modules. Consider the commutative diagram

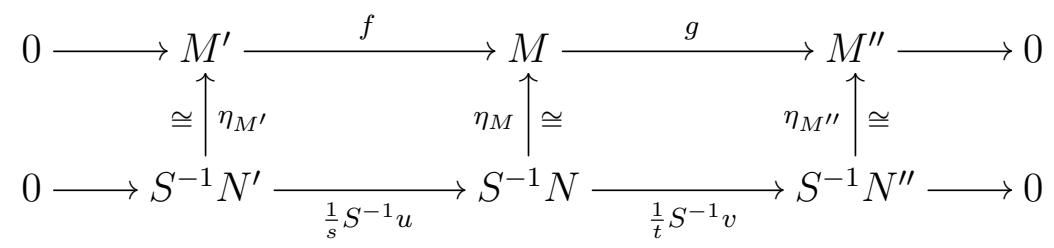

where $\eta_{-}$are the chosen isomorphisms, $s, t \in S, u: N^{\prime} \rightarrow N$ and $v: N \rightarrow N^{\prime \prime}$. We need to show that

$$
\tilde{F}\left(M^{\prime}\right) \stackrel{\tilde{F}(f)}{\longrightarrow} \tilde{F}(M) \stackrel{\tilde{F}(g)}{\longrightarrow} \tilde{F}\left(M^{\prime \prime}\right)
$$

is exact, i.e to show that

$$
S^{-1} F\left(N^{\prime}\right) \stackrel{\frac{1}{s} S^{-1} F(u)}{\longrightarrow} S^{-1} F(N) \stackrel{\frac{1}{t} S^{-1} F(v)}{\longrightarrow} S^{-1} F\left(N^{\prime \prime}\right)
$$

is exact.

Now, if $N_{0}^{\prime \prime}$ is the image of $N$ under the linear map $v: N \rightarrow N^{\prime \prime}$, then we can factor $v$ as $i \circ j$ where $j: N \rightarrow N_{0}^{\prime \prime}$ is surjective and $i: N_{0}^{\prime \prime} \rightarrow N^{\prime \prime}$ is the inclusion map. Let $N_{0}^{\prime}=\operatorname{Ker} j$. Then the sequence

$$
0 \longrightarrow N_{0}^{\prime} \stackrel{k}{\longrightarrow} N \stackrel{j}{\longrightarrow} N_{0}^{\prime \prime} \longrightarrow 0
$$

is exact.

We now have the diagram

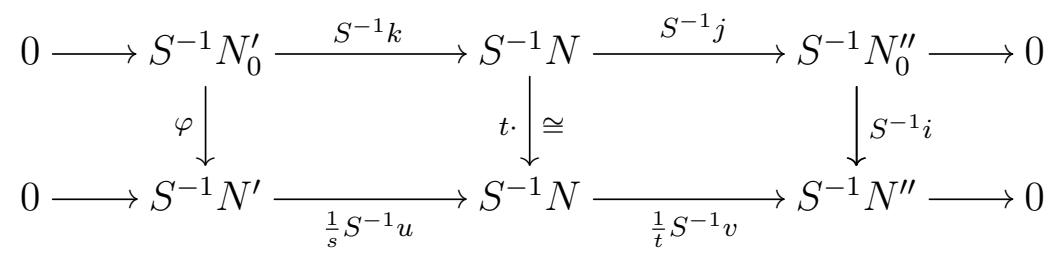

in which the top row is exact after applying $S^{-1}$ to (4) and $\varphi$ is the induced map. Since the inclusion map is injective, $S^{-1} i$ is injective. Hence, the snake lemma shows that $\varphi$ and $S^{-1} i$ are isomorphisms.

Consider the commutative diagram

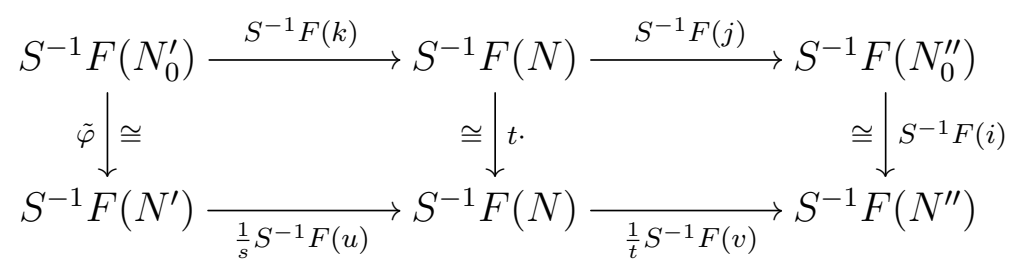


where the vertical arrows are isomorphisms as remarked in 3.1. If the top row is exact, then the bottom row will be exact. But the sequence

$$
F\left(N_{0}^{\prime}\right) \stackrel{F(k)}{\longrightarrow} F(N) \stackrel{F(j)}{\longrightarrow} F\left(N_{0}^{\prime \prime}\right)
$$

is exact since $F$ is half exact. Therefore, the top row is exact and hence $\tilde{F}$ is half exact.

Corollary 3.3. The assignment $\tilde{\Phi}: F \mapsto \tilde{F}$ is an exact functor from $\mathcal{F}_{A}^{f g}$ to $\mathcal{F}_{S^{-1} A}^{f g}$. Moreover, $\Phi$ has the following properties:

i) if $F$ is left exact, then $\tilde{F}$ is also left exact,

ii) if $F$ is right exact, then $\tilde{F}$ is also right exact.

Proof. That $\tilde{\Phi}$ is an exact functor follows from the definition of $\tilde{F}$.

i) Applying the left exact functor $F$ to the exact sequence (4), we get the exact sequence

$$
0 \longrightarrow F\left(N_{0}^{\prime}\right) \stackrel{F(k)}{\longrightarrow} F(N) \stackrel{F(j)}{\longrightarrow} F\left(N_{0}^{\prime \prime}\right) .
$$

Hence,

$$
0 \longrightarrow S^{-1} F\left(N_{0}^{\prime}\right) \stackrel{S^{-1} F(k)}{\longrightarrow} S^{-1} F(N) \stackrel{S^{-1} F(j)}{\longrightarrow} S^{-1} F\left(N_{0}^{\prime \prime}\right)
$$

so that $\tilde{F}$ is left exact.

ii) Similar to i).

Remark 3.4. Let $F \in \mathcal{F}_{A}^{f g}$ and $N \in \mathcal{M}_{A}^{f g}$. The following facts easily follow from the definition of $\tilde{F}$ and repectively using the natural isomorphisms (3) and $S^{-1}\left(N \otimes_{A} N^{\prime}\right) \cong S^{-1} N \otimes_{S^{-1} A} S^{-1} N^{\prime}$.

i) If $F=h_{N}$, then $\tilde{F}=\tilde{h}_{N} \cong h_{S^{-1} N}$.

ii) If $t_{N}=N \otimes_{A}-$ and $F=t_{N}$, then $\tilde{F}=\tilde{t}_{N} \cong t_{S^{-1} N}$.

Proposition 3.5. If $F$ is coherent, then $\tilde{F}$ is also coherent.

Proof. Let $h_{Y} \rightarrow h_{X} \rightarrow F \rightarrow 0$ be a presentation of $F$ as a coherent functor. Then

$$
\tilde{h}_{Y} \rightarrow \tilde{h}_{X} \rightarrow \tilde{F} \rightarrow 0
$$

is exact by 3.3 . Hence, by 3.4 (i) we see that

$$
h_{S^{-1} Y} \rightarrow h_{S^{-1} X} \rightarrow \tilde{F} \rightarrow 0
$$

is a presentation of $\tilde{F}$. Therefore, $\tilde{F}$ is coherent.

Lemma 3.6. Let $F$ be in $\mathcal{C}_{A}^{f g}$. Then $\tilde{F}_{0} \cong(\tilde{F})_{0}$. 
Proof. There is an exact sequence

$$
t_{S^{-1} F(A)} \longrightarrow \tilde{F} \longrightarrow(\tilde{F})_{0} \longrightarrow 0
$$

where $t_{S^{-1} F(A)}=S^{-1} F(A) \otimes_{S^{-1} A}-=\tilde{F}\left(S^{-1} A\right) \otimes_{S^{-1} A}$-. By applying 3.3 to the exact sequence (1), we get the exact sequence

$$
\tilde{t}_{F(A)} \longrightarrow \tilde{F} \longrightarrow \tilde{F}_{0} \longrightarrow 0 \text {. }
$$

Since $\tilde{t}_{F(A)} \cong t_{S^{-1} F(A)}$ by $3.4\left(\right.$ ii) , we get $\tilde{F}_{0} \cong(\tilde{F})_{0}$.

Note 3.7. If $S=A \backslash \mathfrak{p}$ for $\mathfrak{p}$ a prime ideal of $A$, we denote $\tilde{F}$ by $F_{\mathfrak{p}}$. Thus, for any $N \in \mathcal{M}_{A}^{f g}$, we have $F_{\mathfrak{p}}\left(N_{\mathfrak{p}}\right)=F(N)_{\mathfrak{p}}$.

\section{PIDs AND DEDEKInd Domains}

Lemma 4.1. Let $A$ be a noetherian ring and let $F \in \mathcal{F}_{A}^{f g}$ (not necessarily coherent) be half exact. Consider the exact sequence

$$
F(A) \otimes-\stackrel{\alpha}{\longrightarrow} F \longrightarrow F_{0} \longrightarrow 0 .
$$

Then for any $M \in \mathcal{M}_{A}^{f g}$ with projective dimension $\leq 1$, the natural map $\alpha_{M}: F(A) \otimes M \rightarrow F(M)$ is injective.

Proof. Let $M$ be in $\mathcal{M}_{A}^{f g}$. If the projective dimension of $M$ is zero, i.e., $\mathrm{M}$ is projective, then $\alpha_{M}$ is an isomorphism by 2.2 .

Now suppose that $M$ has projective dimension equal to 1 and let

$$
0 \rightarrow P_{1} \rightarrow P_{0} \rightarrow M \rightarrow 0
$$

be a presentation of $M$ with $P_{0}$ and $P_{1}$ projective in $\mathcal{M}_{A}^{f g}$. Then, there is a commutative diagram with $\alpha_{P_{1}}$ and $\alpha_{P_{0}}$ isomorphisms and where $C=\operatorname{Coker}\left(F\left(P_{0}\right) \rightarrow F(M)\right)$.

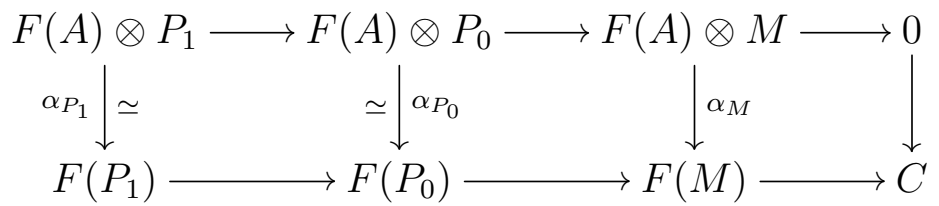

The five lemma shows that $\alpha_{M}$ is injective.

Corollary 4.2. Let $A$ be a noetherian ring of global dimension $\leq 1$ and let $F$ be any half exact functor in $\mathcal{F}_{A}^{f g}$. Then the sequence

$$
0 \longrightarrow F(A) \otimes-\stackrel{\alpha}{\longrightarrow} F \longrightarrow F_{0} \longrightarrow 0
$$

is exact.

Proof. Since $A$ has global dimension $\leq 1$, every finitely generated $A$ module $M$ has projective dimension $\leq 1$ and hence (4.1) applies.

Lemma 4.3. Let $A$ be a PID and let $F \in \mathcal{M}_{A}^{f g}$ be half exact, then $F_{0}$ in (5) is left exact. 
Proof. Let $f: M \rightarrow N$ be an injective homomorphism of finitely generated $A$-modules. We shall prove that $F_{0}(f): F_{0}(M) \rightarrow F_{0}(N)$ is injective. We first reduce to that case that $M$ and $N$ are torsion modules. Since $F_{0}(A)=0$ and hence $F_{0}(L)=0$ for each finitely generated free A-module $L$, there is a commutative diagram,

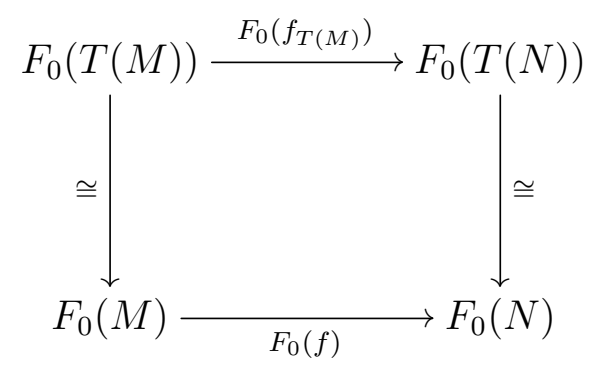

where $T(M)$ denotes the torsion A-submodule of $M$ and the homomorphism $f_{T(M)}: T(M) \rightarrow T(N)$ is induced by $f$. Therefore, we are reduced to the case where $f: M \rightarrow N$ is an injective homomorphism of torsion modules.

We prove the reduced case by induction on the length of the cokernel of $f$. If $l($ Coker $f)=0$, then $f$ is an isomorphism. If $l($ Coker $f)=1$, then $A \bar{y}=$ Coker $f=N / \operatorname{Im} f \cong A /(p)$ for an irreducible element $p$ in $A$ and some $y$ in $N$. Since $p \bar{y}=0$, there is an $x$ in $M$ such that $p y=f(x)$. We first show that the sequence

$$
0 \longrightarrow A \stackrel{g}{\longrightarrow} A \oplus M \stackrel{h}{\longrightarrow} N \longrightarrow 0
$$

is exact; where $g$ and $h$ are defined respectively by $g(a)=(p a, a x)$ and $h((b, z))=f(z)-b y$. First observe that $h$ is surjective since $N=\operatorname{Im} f+A y$. Now, we have

$$
h(g(a))=h(p a, a x)=f(a x)-a p y=a f(x)-a p y=0 .
$$

Also if $(b, z)$ is in the kernel of $h$, then $f(z)=b y$. Thus $b$ belongs to the annihilator of Coker $f$ which is $(p)$. Hence there exists an $a$ in $A$ such that $b=a p$. Therefore

$$
f(z)=b y=a p y=a f(x)=f(a x) .
$$

Since $f$ is injective, we get $z=a x$. Thus $(b, z)=(a p, a x)$ is in the image of $g$ and hence Ker $h=\operatorname{Im} g$. Applying $F_{0}$ to (6) and using the fact that $F_{0}(A)=0$, we see that $F_{0}(f)$ is injective.

Now suppose that $l($ Coker $f)>1$ and that the result is true for all injective homomorphisms $\phi$ such that $l($ Coker $\phi)<l($ Coker $f)$. Take $N^{\prime}$ such that $\operatorname{Im} f \varsubsetneqq N^{\prime} \varsubsetneqq N$ and consider the commutative diagram

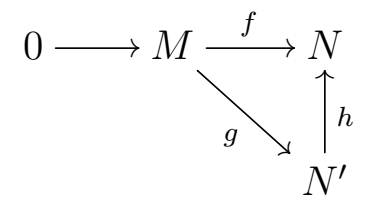


where $h$ is the inclusion map and of course $g$ is injective. Then, $l($ Coker $g)<l($ Coker $f)$ and $l($ Coker $h)<l($ Coker $f)$. Applying $F_{0}$ to $g$ and $h$ and using the induction hypothesis, we see that $F_{0}(g)$ and $F_{0}(h)$ are injective. It follows that $F_{0}(f)$ is injective since it is a composite of injective maps.

We are now ready to extend $[5,6.1]$ to PIDs and Dedekind domains.

Theorem 4.4. Let $A$ be a PID. Then any half exact functor $F \in \mathcal{C}_{A}^{f g}$ is a direct sum of functors of the form

(i) $I=$ the identity functor,

(ii) $\operatorname{Hom}_{A}\left(A / p^{s},-\right)$ and

(iii) $A / p^{s} \otimes-$

where $s \geq 1$ and $p \in A$ is irreducible.

Proof. Let $F$ be a half exact coherent functor and consider the sequence

$$
0 \longrightarrow F(A) \otimes-\stackrel{\alpha}{\longrightarrow} F \longrightarrow F_{0} \longrightarrow 0,
$$

which is exact by 4.2 since the global dimension of a PID is at most one. Then $F_{0}(A)=0$ and $F_{0}$ is a half exact coherent functor. Now by $4.3, F_{0}$ is left exact and hence, by $[5,3.12]$ is isomorphic to $h_{N}$ for some $N \in \mathcal{M}_{A}^{f g}$. Thus $F_{0}$ is a projective object in the category of coherent functors. Therefore, the sequence (7) splits; i.e, $F=(F(A) \otimes-) \oplus F_{0}$.

Since $F_{0}(A)=0$ and $F_{0} \cong h_{N}$ for some $N$ in $\mathcal{M}_{A}^{f g}$, it shows that $N$ is a torsion module and hence $F_{0}$ is a direct sum of functors of the form (ii). Further, decomposing $F(A)$ into direct sums of copies of $A$ and modules of the form $A / p^{s}$, we see that $F(A) \otimes-$ is a direct sum of modules of the form (i) and (iii).

Note that a coherent functor over a PID need not be half exact, see $[5,2.9]$.

Theorem 4.5. Let $A$ be a Dedekind domain and let $F \in \mathcal{C}_{A}^{f g}$ be half exact. Then $F$ is a direct sum of functors of the form

(i) $\operatorname{Hom}_{A}(P,-)$,

(ii) $\operatorname{Hom}_{A}\left(A / \mathfrak{p}^{s},-\right)$ and

(iii) $A / \mathfrak{p}^{s} \otimes-$

where $P$ is a projective $A$-module, $s \geq 1$ and $\mathfrak{p}$ is a non-zero prime ideal in $A$.

Proof. Since a Dedekind domain has global dimension one, by 4.2 , we have a short exact sequence

$$
0 \longrightarrow F(A) \otimes-\stackrel{\alpha}{\longrightarrow} F \longrightarrow F_{0} \longrightarrow 0 .
$$

It now remains to show that $F_{0}$ is left exact, that is, to show that $0 \rightarrow F_{0}(M) \rightarrow F_{0}(N)$ is exact whenever $0 \rightarrow M \rightarrow N$ is exact in $\mathcal{M}_{A}^{f g}$. Towards that goal, let $f_{\mathfrak{p}}: A \rightarrow A_{\mathfrak{p}}$ be the localization map for 
any non-zero prime $\mathfrak{p}$ in $A$. Since $F_{\mathfrak{p}}$ is half exact and coherent on $A_{\mathfrak{p}}$ (which is a DVR), 4.3 shows that

$$
0 \rightarrow\left(F_{\mathfrak{p}}\right)_{0}\left(M_{\mathfrak{p}}\right) \rightarrow\left(F_{\mathfrak{p}}\right)_{0}\left(N_{\mathfrak{p}}\right)
$$

is exact. Since by 3.6 we have $\left(F_{\mathfrak{p}}\right)_{0} \cong\left(F_{0}\right)_{\mathfrak{p}}$, it follows from the definition that $\left(F_{\mathfrak{p}}\right)_{0}\left(M_{\mathfrak{p}}\right)=\left(F_{0}(M)\right)_{\mathfrak{p}}$. Hence,

$$
0 \rightarrow F_{0}(M)_{\mathfrak{p}} \rightarrow F_{0}(N)_{\mathfrak{p}}
$$

is exact for each non-zero prime ideal $\mathfrak{p}$ and therefore, the sequence

$$
0 \rightarrow F_{0}(M) \rightarrow F_{0}(N)
$$

is exact. Thus we have $F_{0} \cong h_{X}$ for some finitely generated $A$-module $X[5,3.12]$. Hence $F_{0}$ is a projective object in $\mathcal{C}_{A}^{f g}$. Therefore, the sequence $(8)$ splits, i.e, $F \cong(F(A) \otimes-) \oplus F_{0}$.

Now, over a Dedekind domain $A$, any finitely generated $A$-module is a direct sum of a projective module and a torsion module. Further, any finitely generated torsion module $M$ is a direct sum of modules of the form $A / \mathfrak{p}^{s}$ where $\mathfrak{p}$ runs through the non-zero prime ideals of $A$ and $s$ is a positive integer $[2,6.3 .20]$. Since $F_{0}(A)=0, X$ is a torsion module and hence $F_{0}$ is a direct sum of functors of the form (ii). Further, decomposing $F(A)$ into a direct sum of a projective module and modules of the form $A / \mathfrak{p}^{s}$ where $s \geq 1$ and $\mathfrak{p}$ is a prime ideal in $A$, we see that $F(A) \otimes-$ is a direct sum of functors of the form (i) and (iii).

ACKNOWLEDGEMENTS. The author would like to acknowledge his supervisor Leif Melkersson for the valuable help and comments on his research and the write up of this paper. He also acknowledges the useful comments and suggestions from the reviewer of this paper which helped to come up with the final version of it. The author also acknowledges the funding received from International Science Program (ISP) through Eastern Africa Universities Mathematics Program (EAUMP) to enable him do his research. He is also thankful to Linköping University and University of Zambia for the opportunity to do research.

\section{REFERENCES}

[1] M. Auslander, Coherent functors. Proc. Conf. Categorical Algebra, Springer (1966) 189-231.

[2] A.J. Berrick and M.E. Keating, An introduction to rings and modules: with $K$-theory in view. Cambridge University press (2000).

[3] V. Franjou and T. Pirashvili, Strict polynomial functors and coherent functors. Manuscripta Mathematica 127 (2008) 23-53.

[4] J. Hall, Openness of versality via coherent functors. Journal für die reine und angewandte Mathematik 722 (2017) 137-182.

[5] R. Hartshorne, Coherent functors. Advances in Mathematics 140 (1998) 44-94.

[6] D.B. Jaffe, Coherent functors, with applications to torsion in the Picard group. Trans. of the Amer. Math. Soc. 349 (1997) 481-527. 
[7] H. Krause, Coherent functors and covariantly finite subcategories. Algebr. Represent. Theory 6 (2003) 475-499.

[8] S. Lang, Algebra. Revised third edition. Springer (2002).

[9] J.J. Rotman, An introduction to homological algebra. Second Edition. Springer (2009).

[10] M. Schlessinger, Functors of Artin rings. Trans. Amer. Math. Soc. 130 (1968) 208-222.

[11] G. Sædén Ståhl, Rees algebras of modules and coherent functors. ArXiv:1409.6464v1 (2014) 